\title{
SOME NEW RESULTS ON THE EICHLER COHOMOLOGY OF AUTOMORPHIC FORMS
}

\author{
BY MARVIN I. KNOPP
}

ABstract. We here formulate and prove several new results concerning the Eichler cohomology of automorphic forms on finitely generated Fuchsian groups of the first kind and, in particular, on $\mathrm{H}$-groups. The Eichler cohomology we introduce is connected with automorphic forms of arbitrary real degree (as opposed to integral degree), with a suitably chosen underlying space of functions analytic in the upper half-plane. We obtain structure theorems for the Eichler cohomology groups which are analogous to earlier results of Eichler and Gunning.

I. Introduction. 1. The purpose of this article is the proof of some new results concerning the Eichler cohomology connected with automorphic forms on finitely generated Fuchsian groups of the first kind (in particular, on $\mathrm{H}$-groups). More specifically we introduce Eichler cohomology groups associated with automorphic forms of arbitrary real (that is, not necessarily integral) degree and, with a suitable underlying space of functions, we determine the structure of these groups. (See Theorems 1 and 2.)

For our present purposes it is sufficient to consider Fuchsian groups $\Gamma$ acting on $\mathscr{H}$, the upper half-plane. That is, $\Gamma$ is a discrete group of linear fractional transformations acting on $\mathscr{H}$. For convenience we normalize $\Gamma$ so that an element $V$ of $\Gamma$ has the form $z \rightarrow(a z+b) /(c z+d)$, with $a, b, c, d$ real and $a d-b c=1$. We also identify $V$ with the matrices $\pm\left(\begin{array}{cc}a & b \\ c & b\end{array}\right)$. In particular we call $\Gamma$ an $H$-group provided

(i) $\Gamma$ is finitely generated,

(ii) $\Gamma$ is discrete, but discontinuous at no point of the real line,

(iii) $\Gamma$ contains translations.

In essence, then, an $H$-group is a finitely generated Fuchsian group of the first kind which has at least one parabolic class.

2. Automorphic forms. The automorphic forms to be considered here are of arbitrary real degree with multiplier system, meromorphic in $\mathscr{H}$, and meromorphic (in the appropriate uniformalizing variables)

An address delivered before the Chicago Meeting of the Society on March 26, 1971 by invitation of the Committee to Select Hour Speakers for Western Sectional Meetings; received by the editors October 25, 1973.

AMS (MOS) subject classifications (1970). Primary 30A58; Secondary 10D15, $20 \mathrm{H} 10$.

Key words and phrases. Automorphic form, H-group, Eichler cohomology group, automorphic integral. 
at all of the parabolic cusps (necessarily finite in number) of a fundamental region $\mathscr{R}$ of $\Gamma$. The characteristic functional equation satisfied by an automorphic form $F$ of degree $r$ and multiplier system $v$, with respect to $\Gamma$, is

$$
\bar{v}(V)(c z+d)^{r} F(V z)=F(z),
$$

for all $V=\left(\begin{array}{cc}a & b \\ c & d\end{array}\right) \in \Gamma$, where $v(V)$ is independent of $z$ and $|v(V)|=1$. From (1.1) follows directly the "consistency condition"

$$
\bar{v}\left(V_{1} V_{2}\right)\left(c_{3} z+d_{3}\right)^{r}=\bar{v}\left(V_{1}\right) \bar{v}\left(V_{2}\right)\left(c_{1} V_{2} z+d_{1}\right)^{r}\left(c_{2} z+d_{2}\right)^{r}
$$

where

and

$$
V_{i}=\left(\begin{array}{ll}
* & * \\
c_{i} & d_{i}
\end{array}\right) \in \Gamma \quad \text { for } i=1,2
$$

$$
V_{1} V_{2}=\left(\begin{array}{ll}
* & * \\
c_{3} & d_{3}
\end{array}\right) .
$$

When $r$ is an integer (2) reduces to $v\left(V_{1} V_{2}\right)=v\left(V_{1}\right) v\left(V_{2}\right)$, for all $V_{1}, V_{2} \in \Gamma$, that is, $v$ is a complex character on the matrix group $\Gamma$. Any function from the matrix group $\Gamma$ into the unit circle of the complex plane which satisfies (1.2) is called a multiplier system for $\Gamma$ of degree $r$.

We introduce the useful stroke operator

$$
\left.F\right|_{v} ^{r} V=\bar{v}(V)(c z+d)^{r} F(V z),
$$

for any function $F$ and $V=\left(\begin{array}{cc}* & * \\ c\end{array}\right) \in \Gamma$. (When there is no risk of confusion we write $F \mid V$ for $\left.F\right|_{v} ^{r} V$.) The functional equation (1.1) may then be written $\left.F\right|_{v} ^{r} V=F$, for $V \in \Gamma$, and the consistency condition (1.2) for $v$ is equivalent to

$$
\left.\left(\left.F\right|_{v} ^{r} V_{1}\right)\right|_{v} ^{r} V_{2}=\left.F\right|_{v} ^{r} V_{1} V_{2}, \quad \text { for } V_{1}, V_{2} \in \Gamma \text {. }
$$

(Both sides of (1.3) equal $F$ if (1.1) holds.)

Suppose $\Gamma$ is an $H$-group and let $S=\left(\begin{array}{ll}1 & \lambda \\ 0 & 1\end{array}\right), \lambda>0$, generate the subgroup $\Gamma_{\infty}$ of translations in $\Gamma$. If $F$ satisfies (1.1), then in particular

$$
F(z+\lambda)=v(S) F(z)=e^{2 \pi i \kappa} F(z),
$$

with $0 \leqq \kappa<1$. Thus, if $F$ is meromorphic in $\mathscr{H}$ and its poles do not accumulate at $\infty, F$ has the Fourier expansion at $\infty$ (actually a Laurent expansion)

$$
F(z)=\sum_{m=-\infty}^{\infty} a_{m} \exp \left\{\frac{2 \pi i(m+\kappa) z}{\lambda}\right\},
$$


valid for $y=\operatorname{Im} z>y_{0}$. Suppose that in addition to $\infty, \Gamma$ has $t \geqq 0$ inequivalent parabolic classes. Each of these classes corresponds to a cyclic subgroup of parabolic elements in $\Gamma$ leaving fixed a parabolic cusp on the boundary of $\mathscr{R}$. Such a parabolic cusp lies on the real axis. (See [15, Chapter 4] for a detailed discussion of the fundamental region $\mathscr{R}$ and its parabolic cusps.) Let $q_{1}, \cdots, q_{t}$ be the inequivalent (with respect to $\Gamma$ ) parabolic cusps (other than $\infty$ ) on the boundary of $\mathscr{R}$ and let $\Gamma_{j}$ be the cyclic subgroup of $\Gamma$ fixing $q_{j}, 1 \leqq j \leqq t$. $\left(\Gamma_{j}\right.$ is called the stabilizer of $q_{j}$ in Г.) Suppose also that

$$
Q_{j}=\left(\begin{array}{ll}
* & * \\
c_{j} & d_{j}
\end{array}\right), \quad 1 \leqq j \leqq t,
$$

is a generator of $\Gamma_{j} ; Q_{j}$ is necessarily parabolic. For $1 \leqq j \leqq t$, put $v\left(Q_{j}\right)=$ $\exp \left(2 \pi i \kappa_{j}\right), 0 \leqq \kappa_{j}<1$. If $F$ satisfies (1.1) with $V=Q_{j}$ and is meromorphic in $\mathscr{H}$ with only finitely many poles in $\mathscr{R}$, then $F$ has the following Fourier expansion at $q_{j}$ :

$$
F(z)=\left(z-q_{j}\right)^{r} \sum_{m=-\infty}^{\infty} a_{m}(j) \exp \left\{\frac{-2 \pi i\left(m+\kappa_{j}\right)}{\lambda_{j}\left(z-q_{j}\right)}\right\},
$$

valid for $y=\operatorname{Im} z>y_{j}$. Here $\lambda_{j}$ is a positive real number called the width of the cusp $q_{j}$ and defined as follows. Let

$$
A_{j}=\left(\begin{array}{cc}
0 & -1 \\
1 & -q_{j}
\end{array}\right),
$$

so that $A_{j}$ has determinant 1 and $A_{j}\left(q_{j}\right)=\infty$. Then $\lambda_{j}>0$ is chosen so that

$$
A_{j}^{-1}\left(\begin{array}{cc}
1 & \lambda_{j} \\
0 & 1
\end{array}\right) A_{j}
$$

generates $\Gamma_{j}$, the stabilizer of $q_{j}$. (See [15, pp. 269-270] for details.)

We are now in a position to give the following

Definition. (a) Suppose $F$ is meromorphic in $\mathscr{H}$ and satisfies the functional equation (1.1) for all $V \in \Gamma$, where $\Gamma$ is a finitely generated Fuchsian group. If $\Gamma$ has parabolic cusps assume, in addition, that $F$ has Fourier expansions (1.4), (1.5) which are finite to the left; that is they contain only finitely many terms with $m<0$. Then $F$ is called an automorphic form of degree $r$ and multiplier system $v$ with respect to $\Gamma$. The set of all such automorphic forms will be denoted by $\{\Gamma, r, v\}$.

(b) Let $F \in\{\Gamma, r, v\}$. Suppose in addition $F$ is holomorphic in $\mathscr{H}$ and has only terms with $m+\kappa \geqq 0$ in (1.4) and $m+\kappa_{j} \geqq 0,1 \leqq j \leqq t$, in (1.5). Then $F$ is called an entire automorphic form. The set of entire automorphic forms in $\{\Gamma, r, v\}$ is denoted $C^{+}(\Gamma, r, v)$. 
(c) If $F \in C^{+}(\Gamma, r, v)$ and has only terms with $m+\kappa>0, m+\kappa_{j}>0$ in the expansions (1.4), (1.5), respectively, then $F$ is called a cusp form. The collection of cusp forms in $\{\Gamma, r, v\}$ is denoted $C^{0}(\Gamma, r, v)$.

It is a well-known fact that if $\Gamma$ is a finitely generated Fuchsian group of the first kind and $r \geqq 0$, then $C^{+}(\Gamma, r, v)=C^{0}(\Gamma, r, v)=\{0\}$ [8], [9]. For Fuchsian groups of the second kind, however, the situation is quite different.

3. Automorphic integrals of integral degree. For the time being suppose that $r$ is an integer $\geqq 0$. A result of G. Bol [1] states that

$$
\frac{d^{r+1}}{d z^{r+1}}\left\{(c z+d)^{r} F(V z)\right\}=(c z+d)^{-r-2} F^{(r+1)}(V z)
$$

for any $V=\left(\begin{array}{cc}a & b \\ c & b\end{array}\right)$, with $a d-b c=1$ and any function $F$ with sufficiently many derivatives. It follows from (1.6) that if $F \in\{\Gamma, r, v\}$, then $F^{(r+1)} \in$ $\{\Gamma,-r-2, v\}$; also if $f \in\{\Gamma,-r-2, v\}$ and $F$ is any $(r+1)$-fold indefinite integral of $f$, then $F$ satisfies the functional equation

$$
\left.F\right|_{v} ^{r} V=F+p_{V}, \quad V \in \Gamma,
$$

where $p_{V}$ is a polynomial in $z$ of degree $\leqq r$. (If $p_{V} \equiv 0$ for all $V \in \Gamma$, then of course $F \in\{\Gamma, r, v\}$.) From (1.7) may be derived the additive analogue of (1.2), a consistency condition for the system of polynomials $p_{V}$ :

$$
p_{V_{1} V_{2}}=p_{V_{1}}{ }_{v}^{r} V_{2}+p_{V_{2}} \text {, for } V_{1}, V_{2} \in \Gamma \text {. }
$$

Definition. (a) If $F$ is a function meromorphic in $\mathscr{H}$ such that $\left.F^{(r+1}\right) \in\{\Gamma,-r-2, v\}$, then $F$ is called an automorphic integral (or Eichler integral) of degree $r$ and multiplier system $v$ with respect to $\Gamma$.

(b) The collection of polynomials $p_{V}$ (necessarily of degree $\leqq r$ ) occurring in (1.7) is called the system of period polynomials of $F$ (or of $\left.F^{(r+1)}\right)$.

We call any collection of polynomials $\left\{p_{V} \mid V \in \Gamma\right\}$, of degree $\leqq r$ which satisfies (1.8) a cocycle (of degree $r$ ). A coboundary (of degree $r$ ) is a cocycle $\left\{p_{V} \mid V \in \Gamma\right\}$ such that $p_{V}=\left.p\right|_{v} ^{r} V-p$ for all $V \in \Gamma$, with $p$ a fixed polynomial of degree $\leqq r$. The parabolic cocycles play a special role in the theory of automorphic integrals; these are the cocycles $\left\{p_{V} \mid V \in \Gamma\right\}$ which satisfy the following additional condition:

Let $Q_{0}=S, Q_{1}, \cdots, Q_{t}$ be a complete set of parabolic (1.9) representatives for $\Gamma$. Then for each $h, 0 \leqq h \leqq t$, there exists a polynomial $p_{h}$ of degree $\leqq r$ such that $p_{Q_{h}}=\left.p_{h}\right|_{v} ^{r} Q_{h}-p_{h}$.

Recall that $S=\left(\begin{array}{ll}1 & 1 \\ 0 & 1\end{array}\right), \lambda>0$, generates the cyclic subgroup $\Gamma_{\infty}$ of translations of $\Gamma$ and that $Q_{h}, 1 \leqq h \leqq t$, generates $\Gamma_{h}$ the stabilizer of $q_{h}$, $1 \leqq h \leqq t$. Here $q_{0}=\infty, q_{1}, \cdots, q_{t}$ are the inequivalent parabolic cusps 
on the boundary of $\mathscr{R}$. Every parabolic element in $\Gamma$ is conjugate in $\Gamma$ to one of the $Q_{h}$.

Definition. (a) The Eichler cohomology group $H_{r, v}^{1}\left(\Gamma, P_{r}\right)$ is defined to be the vector space of cocycles modulo coboundaries. Here $P_{r}$ is the vector space of polynomials of degree $\leqq r$.

(b) Let $\tilde{H}_{r, v}^{1}\left(\Gamma, P_{r}\right)$ be the subgroup of $H_{r, v}^{1}\left(\Gamma, P_{r}\right)$ defined as the space of parabolic cocycles modulo coboundaries.

To $f \in\{\Gamma,-r-2, v\}$ there is attached a unique element of $H_{r, v}^{1}\left(\Gamma, P_{r}\right)$ in the obvious manner: let $F$ be a fixed $(r+1)$-fold integral of $f$ and $\left\{p_{V}\right\}$ the corresponding cocycle determined by (1.7). Any $(r+1)$-fold integral of $f$ has the form $F+p$, with $p \in P_{r}$; then the corresponding cocycle is $\left\{p_{V}+p \mid V-p\right\}$, which determines the same cohomology class as does $\left\{p_{V}\right\}$. Let $\left[p_{V}\right]$ denote the cohomology class of the cocycle $\left\{p_{V}\right\}$. We then define the mapping $\beta$ of $\{\Gamma,-r-2, v\}$ into $H_{r, v}^{1}\left(\Gamma, P_{r}\right)$ by

$$
\beta(f)=\left[p_{V}\right] \text {. }
$$

For $\Gamma$ an $H$-group the structure of $H_{r, v}^{1}\left(\Gamma, P_{r}\right)$ and $\tilde{H}_{r, v}^{1}\left(\Gamma, P_{r}\right)$ has been completely determined in [2], [5], [6] and [13]. (See also [11], [12], [16] and [10, Chapter 5], where the structure of these groups is determined in the more general situation when $\Gamma$ is a finitely generated Kleinian group.) The results are as follows.

Theorem A. The vector spaces $C^{0}(\Gamma,-r-2, \bar{v}) \oplus C^{+}(\Gamma,-r-2, v)$ and $H_{r, v}^{1}\left(\Gamma, P_{r}\right)$ are canonically isomorphic. Under the same mapping $C^{0}(\Gamma,-r-2, \bar{v}) \oplus C^{0}(\Gamma,-r-2, v)$ is isomorphic to $\tilde{H}_{r, v}^{1}\left(\Gamma, P_{r}\right)$.

REMARKS. (a) With $r$ an integer and $v$ a multiplier system of degree $-r-2, \bar{v}$ is likewise a multiplier system of degree $-r-2$.

(b) Theorem A shows that $H_{r, v}^{1}\left(\Gamma, P_{r}\right)$ is a finite dimensional complex vector space, since $C^{+}$and $C^{0}$ are of finite dimension.

(c) Theorem A was first proved by Gunning [5] with $\Gamma$ a finitely generated Fuchsian group of the first kind, not necessarily an $H$-group, but with the mild restriction that $v$ is always a root of unity. Gunning states the result in terms of the existence of exact sequences of the form

$$
\begin{aligned}
& 0 \rightarrow C^{+}(\Gamma,-r-2, v) \rightarrow H_{r, v}^{1}\left(\Gamma, P_{r}\right) \rightarrow C^{0}(\Gamma,-r-2, \bar{v}) \rightarrow 0, \\
& 0 \rightarrow C^{0}(\Gamma,-r-2, v) \rightarrow \tilde{H}_{r, v}^{1}\left(\Gamma, P_{r}\right) \rightarrow C^{0}(\Gamma,-r-2, \bar{v}) \rightarrow 0 .
\end{aligned}
$$

These results originate with Eichler [2], who, however, dealt only with $\tilde{H}_{r, v}^{1}$, under the additional (but inessential) restriction that $r$ be even and $v$ be $\equiv 1$. The proofs of Theorem A given in [6] and [13] are valid for arbitrary integral $r$ and arbitrary multiplier system $v$.

(d) The proof of Theorem A given in [6] proceeds by explicitly constructing a linear mapping

$$
\mu: C^{0}(\Gamma-r-2, \bar{v}) \oplus C^{+}(\Gamma,-r-2, v) \rightarrow H_{r, v}^{1}\left(\Gamma, P_{r}\right)
$$


of the form $\mu(f, g)=\alpha(f)+\beta(g)$, where $f \in C^{0}(\bar{v}), g \in C^{+}(v)$, and $\beta$ is defined by (1.10). The definition of $\alpha$ is more complicated and based upon the notion of the "supplementary series" introduced in [7]. The construction of $\alpha$ also can be carried out using the more recent results of Douglas Niebur [18], as we shall observe later. The proof that $\mu$ is 1-1 follows from results of [7]; the proof in [6] that $\mu$ is onto follows Eichler [2, pp. 274-276], utilizing a generalized Riemann-Roch theorem due to Petersson [23, Theorem 9]. Lehner's treatment of Theorem A differs from that of [6] in the proof that $\mu$ is onto, applying the Generalized Poincare Series (see §II.1) rather than the Riemann-Roch theorem.

4. Eichler cohomology of arbitrary real degree. Now let $r$ be an arbitrary real number and $v$ a multiplier system for $\Gamma$ of degree $r$. If $\mathscr{F}$ is any vector space of functions preserved under the stroke operation $\left.\right|_{v} ^{r}$, one may form the cohomology groups $H_{r, v}^{1}(\Gamma, \mathscr{F})$ and $\tilde{H}_{r, v}^{1}(\Gamma, \mathscr{F})$ in complete analogy to the above construction of $H_{r, v}^{1}\left(\Gamma, P_{r}\right)$ and $\tilde{H}_{r, v}^{1}\left(\Gamma, P_{r}\right)$. Of particular interest here will be the groups formed with $\mathscr{F}=\mathscr{P}$, where $\mathscr{P}$ is the space of functions $g$ holomorphic in $\mathscr{H}$ which satisfy the growth condition

$$
|g(z)|<K\left(|z|^{\rho}+y^{-\sigma}\right), \quad y=\operatorname{Im} z>0,
$$

for some positive constants $K, \rho$ and $\sigma$. It is easy to verify that $\mathscr{P}$ is preserved under $\left.\right|_{v} ^{r}$ for any real $r$ and any $H$-group $\Gamma$ and also under differentiation and anti-differentiation. Note that $\mathscr{P}$ is a ring as well as an infinite dimensional complex vector space, containing all polynomials and all entire automorphic forms with respect to any $H$-group (see $[15$, p. 281] and [8]).

We now extend the notion of automorphic integral to arbitrary real degree.

Definition. If $F$ is a function meromorphic in $\mathscr{H}$ such that

$$
\left.F\right|_{v} ^{r} V-F \in \mathscr{P}, \text { for } V \in \Gamma \text {, }
$$

and for each $j, 1 \leqq j \leqq t$, there exists an integer $m_{j}$ such that $\exp \left\{2 \pi i\left(m_{j}+\kappa_{j}\right) / \lambda_{j}\left(z-q_{j}\right)\right\} F(z)$ has a limit as $z \rightarrow q_{j}$ within $\mathscr{R}$ and also there exists an integer $m_{0}$ such that $\exp \left\{-2 \pi i\left(m_{0}+\kappa\right) z \mid \lambda\right\} F(z)$ has a limit as $z \rightarrow i \infty$ within $\mathscr{R}$, then we call $F$ an automorphic integral of degree $r$ with respect to $\Gamma$.

REMARKS. If $r$ is an integer $\geqq 0$ and $\mathscr{P}$ is replaced by $P_{r}$ in (1.12), then this definition coincides with our previous definition of automorphic (or Eichler) integral. Niebur's definition of automorphic integral is somewhat more restrictive than that given here. If (1.12) holds then $\left\{\left.F\right|_{v} ^{r} V-F\right\}$ is clearly a cocycle in $\mathscr{P}$ of degree $r$ for the group $\Gamma$. In (1.12) one can of course replace $\mathscr{P}$ by any space $\mathscr{F}$ of functions meromorphic in $\mathscr{H}$ and 
obtain cocycles of degree $r$ lying in $\mathscr{F}$. We refer to the cocycle $\left\{F \mid{ }_{v}^{r} V-F\right\}$ as the cocycle of period functions of the automorphic integral $F$.

By considering automorphic integrals of arbitrary real degree, we here determine the structure of both $H_{r, v}^{1}(\Gamma, \mathscr{P})$ and $\tilde{H}_{r, v}^{1}(\Gamma, \mathscr{P})$ for real $r \geqq 0$ and real $r \leqq-2$ with an arbitrary multiplier system of degree $r$ and $\Gamma$ an $H$-group. Specifically, we prove the following.

THEOREM 1. If $r \geqq 0$ or $r \leqq-2$ with $v$ a multiplier system of degree $r$, then $C^{0}(\Gamma,-r-2, \bar{v})$ is isomorphic to $\tilde{H}_{r, v}^{1}(\Gamma, \mathscr{P})$ under a canonical isomorphism $\alpha$.

THEOREM 2. Under the same conditions we have

$$
H_{r, v}^{1}(\Gamma, \mathscr{P}) \cong{ }_{\alpha} C^{0}(\Gamma,-r-2, \bar{v})
$$

REMARKS. (a) For $r \leqq-2,-r-2 \geqq 0$, so that $C^{0}(\Gamma,-r-2, \bar{v})=\{0\}$; hence $H_{r, v}^{1}(\Gamma, \mathscr{P})=\tilde{H}_{r, v}^{1}(\Gamma, \mathscr{P})=\{0\}$ in this case.

(b) For $r \geqq 0$ the mapping $\alpha$ is the one mentioned above in connection with Theorem A. We give the definition of $\alpha$ in $\S I I I .2(r>0)$ and $\S I I I .3$ $(r=0)$.

(c) We first prove Theorem 1 and later prove a general result (Proposition 9) about the space $\mathscr{P}$, from which it is possible to show that for any real degree $r$ and corresponding multiplier system $v$ every cocycle in $\mathscr{P}$ is parabolic. Thus

$$
H_{r, v}^{1}(\Gamma, \mathscr{P})=\tilde{H}_{r, v}^{1}(\Gamma, \mathscr{P})
$$

holds for arbitrary real $r$; Theorem 2 follows from Theorem 1 and (1.13). I am grateful to B. A. Taylor, who supplied the proof of Proposition 9.

For arbitrary real $r$ with corresponding multiplier system $v, \bar{v}$ is a multiplier system of degree $-r-2$. Thus we are permitted to speak of $C^{0}(\Gamma,-r-2, \bar{v})$.

II. The Generalized Poincaré Series. 1. The proof of Theorem 1 is based upon the more fundamental

THEOREM 3. Let $r$ be any real number and $v$ a multiplier system of degree $r$. Suppose $\left\{\varphi_{V} \mid V \in \Gamma\right\}$ is a parabolic cocycle of degree $r$ in $\mathscr{P}$; that is, $\varphi_{V} \in \mathscr{P}, \varphi_{V_{1} V_{2}}=\left.\varphi_{V_{1}}\right|_{v} ^{r} V_{2}+\varphi_{V_{2}}$, for all $V_{1}, V_{2} \in \Gamma$, and

$$
\begin{aligned}
& \text { for each } j \text { such that } 0 \leqq j \leqq t, \text { there exists } \varphi_{j} \in \mathscr{P} \text { such that } \\
& \varphi_{Q_{j}}=\left.\varphi_{j}\right|_{v} ^{r} Q_{j}-\varphi_{j} .
\end{aligned}
$$


Then there exists a function $\Phi$, holomorphic in $\mathscr{H}$, such that

$$
\Phi \mid{ }_{v}^{r} V-\Phi=\varphi_{V}, \text { for all } V \in \Gamma,
$$

and with expansions at the parabolic cusps $q_{j}, 0 \leqq j \leqq t$, of the form

$$
\begin{aligned}
& \Phi(z)=\varphi_{j}(z)+\left(z-q_{j}\right)^{r} \sum_{m=-m j}^{\infty} a_{m}(j) \exp \left\{\frac{-2 \pi i\left(m+\kappa_{j}\right)}{\lambda_{j}\left(z-q_{j}\right)}\right\}, \\
& \Phi(z)=\varphi_{0}(z)+\sum_{m=-m_{0}}^{\infty} a_{m}(0) \exp \left\{\frac{2 \pi i(m+\kappa) z}{\lambda}\right\}, \quad j=0 .
\end{aligned}
$$

(Compare (2.3) with (1.4) and (1.5); recall that $q_{0}=\infty, Q_{0}=S$.) The functions $\varphi_{V}$ may be called the period functions of $\Phi$.

The construction of the function $\Phi$ of Theorem 3 involves the introduction of a "Generalized Poincaré Series," first discussed by Eichler $[3],[4]$, discovered independently somewhat later by the author, and more recently applied by Lehner to Kleinian as well as Fuchsian groups [13], [14], [16].

DEFINITION. Suppose $\left\{\varphi_{V}\right\}$ is a parabolic cocycle of degree $V$ which satisfies the additional condition that $\varphi_{S}=0$. Suppose $k$ is a "large" positive even integer and $w$ is a multiplier system for $\Gamma$ and the degree $-k$. Then the Generalized Poincaré Series $\Psi\left(\left\{\varphi_{V}\right\}, k, w ; z\right)=\Psi(z)$ is defined by

$$
\Psi(z)=\sum_{V \in \mathscr{M}} \varphi_{V}(z) \bar{w}(V)(c z+d)^{-k}
$$

where $\mathscr{M}$ is a complete set of coset representatives for $\Gamma / \Gamma_{\infty}$ and $V=\left(\begin{array}{ll}* & * \\ c & { }_{d}^{*}\end{array}\right)$. (Recall that $\Gamma_{\infty}$ is the stabilizer of $\infty$ in $\Gamma$; it is the cyclic group generated by $S$.)

REMARKS. 1. We shall show below that for $k$ sufficiently large, the series (2.4) converges, in fact uniformly on compact subsets of $\mathscr{H}$. Indeed it will follow that, for such $k, \Psi \in \mathscr{P}$.

2. The summation in (2.4) is really over all distinct lower rows $c, d$ of elements of $\Gamma$.

3. The assumption $\varphi_{S}=0$ has been made to insure that the individual terms of the series (2.4) are independent of the choice $V$ of coset representatives. If $V, V^{\prime}$ lie in the same coset of $\Gamma / \Gamma_{\infty}$, then $V, V^{\prime}$ have the same lower row $c, d$ and $V^{\prime}=S^{t} V$, where $t$ is an integer. Then

$$
\varphi_{V^{\prime}}=\varphi_{S^{t} V}=\varphi_{S^{t}} \mid V+\varphi_{V}
$$


but $\varphi_{S}=0$ implies $\varphi_{S^{t}}=0$, so that $\varphi_{V^{\prime}}=\varphi_{V}$ and the desired independence follows.

4. On the assumption that $k$ is sufficiently large we shall show that the series in (2.4) converges absolutely and it follows readily that, with $M=\left(\begin{array}{ll}* & * \\ \gamma & \delta\end{array}\right) \in \Gamma$,

$$
\left.\Psi\right|_{v} ^{r} M=w(M)(\gamma z+\delta)^{k} \Psi(z)-w(M)(\gamma z+\delta)^{k} g(z) \varphi_{M}(z),
$$

where $g(z)$ is the Eisenstein series

$$
g(z)=\sum_{V \in \mathscr{M}} \bar{w}(V)(c z+d)^{-k} .
$$

The functional equation (2.5) is a straightforward consequence of the absolute convergence of (2.4), the consistency condition for the cocycle $\left\{\varphi_{V}\right\}$ :

$$
\varphi_{V_{1} V_{2}}=\varphi_{V_{1}} \mid{ }_{v}^{r} V_{2}+\varphi_{V_{2}} \quad\left(V_{1}, V_{2} \in \Gamma\right),
$$

and the consistency condition for the multiplier system $w$ :

$$
\begin{array}{r}
\bar{w}\left(V_{1} V_{2}\right)\left(c_{3} z+d_{3}\right)^{-k}=\bar{w}\left(V_{1}\right) \bar{w}\left(V_{2}\right)\left(c_{1} V_{2} z+d_{1}\right)^{-k}\left(c_{2} z+d_{2}\right)^{-k} \\
\left(V_{1}, V_{2} \in \Gamma\right)
\end{array}
$$

For $k$ sufficiently large there is absolute convergence of the series in (2.6) and it follows that

$$
g(M z)=w(M)(\gamma z+\delta)^{k} g(z),
$$

for $M=\left(\begin{array}{cc}* & * \\ \gamma & \delta\end{array}\right) \in \Gamma$. Thus, putting $F(z)=-\Psi(z) / g(z)$ and applying (2.5); we find that

$$
\begin{aligned}
F \mid{ }_{v}^{r} M & =-\left(\Psi \mid{ }_{v}^{r} M\right) / g(M z) \\
& =-\Psi(z) / g(z)+\varphi_{M}(z)=F(z)+\varphi_{M}(z),
\end{aligned}
$$

so that $F$ is a solution of the functional equation (2.2). Indeed $F$ has expansions of the type (2.3) at the parabolic cusps, but may have poles in $\mathscr{H}$; thus it may have to be modified in order to obtain the function $\Phi$ of Theorem 3.

2. Estimation of the cocycle. The proof of absolute convergence of the series defining $\Psi(z)$ is based upon a series of lemmas.

LEMMA 4. For real numbers $c, d$ and $z=x+i y$, we have

$$
\left(\frac{y^{2}}{1+4|z|^{2}}\right)\left(c^{2}+d^{2}\right) \leqq|c z+d|^{2} \leqq 2\left(|z|^{2}+y^{-2}\right)\left(c^{2}+d^{2}\right) .
$$

REMARK. This is essentially Lemma 4 of [14]. 
Proof. Schwarz's inequality gives $|c z+d|^{2} \leqq\left(|z|^{2}+1\right)\left(c^{2}+d^{2}\right)$. Since $|z|^{2}+1 \leqq 2\left(|z|^{2}+y^{-2}\right)$, the upper bound follows. To obtain the lower bound observe that $|c z+d|^{2} \geqq c^{2} y^{2}$ and $|z|^{2}|c z+d|^{2}=\left.|c| z\right|^{2}+\left.d z\right|^{2} \geqq d^{2} y^{2}$, so that

$$
|c z+d|^{2} \geqq y^{2}\left(c^{2}+d^{2}\right) /\left(1+|z|^{2}\right) \geqq y^{2}\left(c^{2}+d^{2}\right) /\left(1+4|z|^{2}\right) .
$$

Suppose $Q_{0}, \cdots, Q_{t}, V_{1}, \cdots, V_{s}$ is a fixed set of generators of $\Gamma$, including the $t+1$ parabolic generators $Q_{0}, \cdots, Q_{t}$ and the nonparabolic generators $V_{1}, \cdots, V_{s}$. If $A \in \Gamma$ consider a factorization of $A$ into sections (see [14, pp. 156-157]), $A=C_{1} \cdots C_{\alpha}$. Each section $C_{i}$ is either a nonparabolic generator of $\Gamma$ or a power of a parabolic generator of $\Gamma$. The importance of this factorization into sections lies in the result of Eichler [3, Theorem 1] that, for any $A \in \Gamma$, the factorization can be carried out so that

$$
q \leqq m_{1} \log \mu(A)+m_{2},
$$

where $m_{1}, m_{2}>0$ are independent of $A$ and

$$
\mu(A)=a^{2}+b^{2}+c^{2}+d^{2} \text { if } A=\left(\begin{array}{ll}
a & b \\
c & d
\end{array}\right) .
$$

(Note that $\mu(A) \geqq 2$ and $\mu(A B) \leqq \mu(A) \mu(B)$ for matrices $A$ and $B$ of determinant 1.)

We assume, as we may, that the cocycle $\left\{\varphi_{V}\right\}$ in $\mathscr{P}$ satisfies

$$
\begin{aligned}
\left|\varphi_{V_{i}}(z)\right|<K\left(|z|^{\rho}+y^{-\sigma}\right), & \text { for } 1 \leqq i \leqq s, \\
\left|\varphi_{j}(z)\right|<K\left(|z|^{\rho}+y^{-\sigma}\right), & \text { for } 0 \leqq j \leqq t .
\end{aligned}
$$

Here $\varphi_{j}(z)$ is defined by (2.1) and $K, \rho, \sigma$ are positive constants independent of the particular generator involved. Assume also $2 \sigma>-r, \rho>r$.

Lemma 5. If $\left\{\varphi_{V}\right\}$ is a parabolic cocycle then there exists $K^{*}>0$ depending only upon $\Gamma$ and $\left\{\varphi_{V}\right\}$ such that

$$
\left|\varphi_{C_{h}}\right|_{v}^{r} C_{h+1} \cdots C_{q} \mid \leqq K^{*} \mu(A)^{e}\left\{|z|^{6 e-2 r}+y^{-6 e+2 r}\right\},
$$

for $1 \leqq h \leqq q$. Here $e=\max (\rho / 2, \sigma+r / 2)$ and $A=C_{1} \cdots C_{q}$ is a factorization into sections of $A \in \Gamma$.

Proof. The proof follows that of Theorem 1 in [14]. Consider first 
the case when $C_{h}$ is a nonparabolic generator. Let $V=C_{h+1} \cdots C_{q}=$ $\left(\begin{array}{ll}\alpha & \beta \\ \gamma & \delta\end{array}\right)$. Then, by (2.11),

By Lemma 4,

$$
\begin{aligned}
\left|\varphi_{C_{h}}\right| V \mid & =|\gamma z+\delta|^{r}\left|\varphi_{C_{h}}(V z)\right| \\
& <|\gamma z+\delta|^{r} \cdot K\left\{|V z|^{\rho}+y^{-\sigma}|\gamma z+\delta|^{2 \sigma}\right\} \\
& =K|\alpha z+\beta|^{\rho}|\gamma z+\delta|^{r-\rho}+K|\gamma z+\delta|^{2 \sigma+r} y^{-} .
\end{aligned}
$$

$$
\begin{aligned}
|\alpha z+\beta|^{\rho} & \leqq 2^{\rho / 2}\left(|z|^{2}+y^{-2}\right)^{\rho / 2}\left(\alpha^{2}+\beta^{2}\right)^{\rho / 2} \\
|\gamma z+\delta|^{2 \sigma+r} & \leqq 2^{\sigma+r / 2}\left(|z|^{2}+y^{-2}\right)^{\sigma+r / 2}\left(\gamma^{2}+\delta^{2}\right)^{\sigma+r / 2}
\end{aligned}
$$

and

Hence

$$
|\gamma z+\delta|^{r-\rho} \leqq\left(y^{2} /\left(1+4|z|^{2}\right)\right)^{(r-\rho) / 2}\left(\gamma^{2}+\delta^{2}\right)^{(r-\rho) / 2} .
$$

$$
\begin{aligned}
\left|\varphi_{C_{h}}\right| V \mid< & K 2^{\rho / 2}\left(|z|^{2}+y^{-2}\right)^{\rho / 2}\left(\alpha^{2}+\beta^{2}\right)^{\rho / 2} \\
& \cdot\left(\frac{1+4|z|^{2}}{y^{2}}\right)^{(\rho-r) / 2}\left(\gamma^{2}+\delta^{2}\right)^{(r-\rho) / 2} \\
& +K 2^{\sigma+r / 2}\left(|z|^{2}+y^{-2}\right)^{\sigma+r / 2}\left(\gamma^{2}+\delta^{2}\right)^{\sigma+r / 2} y^{-\sigma} .
\end{aligned}
$$

Since the nonzero $\gamma,\left(\begin{array}{ll}* & * \\ \gamma & *\end{array}\right) \in \Gamma$, with $\Gamma$ discrete, have a positive lower bound, it follows that $\gamma^{2}+\delta^{2}$ has a positive lower bound; hence

$$
\begin{aligned}
\left|\varphi_{C_{h}}\right| V \mid< & K_{1}\left(\alpha^{2}+\beta^{2}\right)^{\rho / 2}\left(|z|^{2}+y^{-2}\right)^{\rho / 2}\left(\frac{1+4|z|^{2}}{y^{2}}\right)^{(\rho-r) / 2} \\
& +K_{1}^{\prime}\left(\gamma^{2}+\delta^{2}\right)^{\sigma+r / 2}\left(|z|^{2}+y^{-2}\right)^{\sigma+r / 2} y^{-\sigma}
\end{aligned}
$$

By [3, Theorem 2]

$$
\alpha^{2}+\beta^{2}+\gamma^{2}+\delta^{2}=\mu(V) \leqq K_{2} \mu(A),
$$

so that

$$
\begin{aligned}
\left|\varphi_{C_{h}}\right| V \mid \leqq & K_{3} \mu(A)^{\rho / 2}\left(|z|^{2}+y^{-2}\right)^{\rho / 2} y^{r-\rho}\left(1+4|z|^{2}\right)^{(\rho-r) / 2} \\
& +K_{3}^{\prime} \mu(A)^{\sigma+r / 2} y^{-\sigma}\left(|z|^{2}+y^{-2}\right)^{\sigma+r / 2}
\end{aligned}
$$

Letting $e=\max (\rho / 2, \sigma+r / 2)$, we have

$$
\begin{aligned}
\left|f_{C_{h}}\right| V \mid & \leqq K_{4} \mu(A)^{e}\left(|z|^{2}+y^{-2}\right)^{e}\left\{y^{r-\rho}\left(1+4|z|^{2}\right)^{(\rho-r) / 2}+y^{-\sigma}\right\} \\
& \leqq K_{4} \mu(A)^{e}\left(|z|^{2}+y^{-2}\right)^{e}\left\{\frac{1}{2} y^{2 r-2 \rho}+\frac{1}{2}\left(1+4|z|^{2}\right)^{\rho-r}+y^{-\sigma}\right\} .
\end{aligned}
$$

Now $\sigma \leqq e-r / 2$ and $\rho-r \leqq 2 e-r$, so that

$$
\begin{aligned}
\left|f_{C_{h}}\right| V \mid & \leqq K_{5} \mu(A)^{e}\left(|z|^{2}+y^{-2}\right)^{e}\left\{|z|^{4 e-2 r}+y^{-4 e+2 r}\right\} \\
& \leqq K_{6} \mu(A)^{e}\left(|z|^{6 e-2 r}+y^{-6 e+2 r}\right) .
\end{aligned}
$$


We now deal with the case in which $C_{h}$ is a parabolic section, that is $C_{h}=Q_{j}^{m}$, with $j=j(h)$ between 0 and $t$. Then

$$
\varphi_{Q_{j}}=\varphi_{j} \mid Q_{j}-\varphi_{j}
$$

and therefore, by (2.7), also

From this it follows that

$$
\varphi_{C_{h}}=\varphi_{j} \mid C_{h}-\varphi_{j}
$$

$$
\varphi_{C_{h}}\left|C_{h+1} \cdots C_{q}=\varphi_{j}\right| C_{h} \cdots C_{q}-\varphi_{j} \mid C_{h+1} \cdots C_{\alpha} .
$$

The previous argument applies to each of the two terms on the righthand side to yield

$$
\left|\varphi_{C_{h}}\right| C_{h+1} \cdots C_{q} \mid \leqq K_{7} \mu(A)^{e}\left(|z|^{6 e-2 r}+y^{-6 e+2 r}\right) .
$$

The proof is complete.

It is helpful at this point to introduce a specific fundamental region; we shall employ the Ford fundamental region $\mathscr{R}$ defined as follows (see $[17$, p. 58$]$ or $[15$, p. 139]):

$$
\begin{aligned}
& \mathscr{R}=\{z \in \mathscr{H}|| \operatorname{Re} z \mid<\lambda / 2 \text { and }|c z+d|>1 \\
& \left.\qquad \text { for all } V=\left(\begin{array}{ll}
* & * \\
c & d
\end{array}\right) \in \Gamma-\Gamma_{\infty}\right\} .
\end{aligned}
$$

(Please note that the definition of $\mathscr{R}$ is misprinted in [17].) Then there exists $y_{0}>0$ with $i y_{0} \in \mathscr{R}$. Now determine $\mathscr{M}$ by the condition that $A \in \mathscr{M}$ if $-\lambda / 2 \leqq \operatorname{Re}\left\{A\left(i y_{0}\right)\right\}<\lambda / 2$. (Note that $\mathscr{M} \cap \Gamma_{\infty}=\{ \pm I\}$.)

LeMmA 6. If $A=\left(\begin{array}{ll}a & b \\ c & d\end{array}\right) \in \mathscr{M}$, chosen as indicated above, then

$$
\mu(A) \leqq K^{\prime}\left(c^{2}+d^{2}\right),
$$

for $K^{\prime}>0$, independent of $A$.

Proof. $A \in \mathscr{M}$ implies $\left|\operatorname{Re} A\left(i y_{0}\right)\right| \leqq \lambda / 2$. Also,

$$
0<\operatorname{Im} A\left(i y_{0}\right)=y_{0}\left|c i y_{0}+d\right|^{-2}=y_{0}\left(c^{2} y_{0}^{2}+d^{2}\right)^{-1} \text {. }
$$

If $c=0$, then $d=1$ and $\operatorname{Im} A\left(i y_{0}\right)=y_{0}$. If $c \neq 0$, then $|c|>m_{3}$, so that

$$
\operatorname{Im} A\left(i y_{0}\right)=y_{0} /\left(c^{2} y_{0}^{2}+d^{2}\right) \leqq y_{0} / c^{2} y_{0}^{2}<1 / m_{3}^{2} y_{0} .
$$

Hence $\left|A\left(i y_{0}\right)\right|<K_{4}^{\prime}$; that is, $\left|\left(a\left(i y_{0}\right)+b\right) /\left(c\left(i y_{0}\right)+d\right)\right|<K_{4}^{\prime}$. From this we conclude that $a^{2} y_{0}^{2}+b^{2}<K_{4}^{\prime \prime}\left(c^{2} y_{0}^{2}+d^{2}\right)$ and hence $a^{2}+b^{2}<K_{5}^{\prime}\left(c^{2}+d^{2}\right)$. The result follows since $\mu(A)=a^{2}+b^{2}+c^{2}+d^{2}$.

3. Convergence of the Generalized Poincaré Series. Suppose $A \in \mathscr{M}$. As before write $A=C_{1} \cdots C_{q}$, a product of sections. Applying (2.7) 
repeatedly we find that

$$
\varphi_{A}=\varphi_{C_{1} \cdots C_{q}}=\varphi_{C_{1}}\left|C_{2} \cdots C_{q}+\varphi_{C_{2}}\right| C_{3} \cdots C_{q}+\cdots+\varphi_{C_{q}},
$$

with $q \leqq m_{1} \log \mu(A)+m_{2}$ terms on the right-hand side. We need to estimate the absolute value of the general term of the series (2.4). This is

$$
\left|\varphi_{A}(z) \bar{w}(A)(c z+d)^{k}\right|=\left|\varphi_{A}(z)\right||c z+d|^{-k} .
$$

By (2.13) and Lemma 5, we have

$$
\left|\varphi_{A}(z)\right| \leqq K^{*} \mu(A)^{e}\left(|z|^{\eta}+y^{-\eta}\right) q \leqq K_{1}^{*} \mu(A)^{e+1}\left(|z|^{\eta}+y^{-\eta}\right),
$$

where $\eta=6 e-2 r$ and we have used $q \leqq m_{1} \log \mu(A)+m_{2} \leqq m_{3} \mu(A)$. Lemma 6 yields

$$
\left|\varphi_{A}(z)\right| \leqq K_{2}^{*}\left(c^{2}+d^{2}\right)^{e+1}\left(|z|^{\eta}+y^{-\eta}\right),
$$

and, by Lemma 4 ,

Hence,

$$
\left|\varphi_{A}(z)\right| \leqq K_{2}^{*}|c z+d|^{2 e+2}\left(\frac{1+4|z|^{2}}{y^{2}}\right)^{e+1}\left(|z|^{\eta}+y^{-\eta}\right) .
$$

$$
\left|\varphi_{A}(z)\right||c z+d|^{-k} \leqq K_{2}^{*}|c z+d|^{2 e+2-k}\left(\frac{1+4|z|^{2}}{y^{2}}\right)^{e+1}\left(|z|^{\eta}+y^{-\eta}\right) .
$$

With $k>2 e+4$ it follows that $k-2 e-2>2$, hence by [15, pp. 276-277] that (2.4) converges absolutely and, in fact, uniformly on compact subsets of $\mathscr{H}$.

Proposition 7. For $k$ sufficiently large $(>2 e+4)$ the Generalized Poincaré Series (2.4) converges absolutely and, in fact, $\Psi \in \mathscr{P}$.

Proof. The absolute convergence and the fact that $\Psi$ is holomorphic in $\mathscr{H}$ have already been proved. To show that $\Psi \in \mathscr{P}$ apply Lemma 4 to (2.14) to get

$$
\begin{aligned}
\left|\varphi_{A}(z)\right||c z+d|^{-k} & \leqq K_{2}^{*}\left(c^{2}+d^{2}\right)^{e+1-k / 2}\left(\frac{1+4|z|^{2}}{y^{2}}\right)^{k / 2}\left(|z|^{\eta}+y^{-\eta}\right) \\
& =K_{2}^{*}|c i+d|^{2 e+2-k}\left(\frac{1+4|z|^{2}}{y^{2}}\right)^{k / 2}\left(|z|^{\eta}+y^{-\eta}\right) .
\end{aligned}
$$

The convergence of $\sum_{A \in \mathscr{M}}|c i+d|^{2 e+2-k}$ implies that $\Psi \in \mathscr{P}$.

4. Proof of Theorem 3. Let $\left\{\varphi_{V}\right\}$ be a parabolic cocycle in $\mathscr{P}$ for $\Gamma$ of degree $r$ and multiplier system $v$. For $V \in \Gamma$, put

$$
\varphi_{V}^{*}=\varphi_{V}-\left(\left.\varphi_{0}\right|_{v} ^{r} V-\varphi_{0}\right)
$$


Then $\left\{\varphi_{V}^{*}\right\}$ is again a parabolic cocycle in $\mathscr{P}$ and now $\varphi_{S}^{*}=\varphi_{0} \mid S-\varphi_{0}-$ $\left(\varphi_{0} \mid S-\varphi_{0}\right)=0$. Thus we may form the Generalized Poincaré Series $\Psi\left(\left\{\varphi_{V}^{*}\right\}, k, w ; z\right)=\Psi^{*}(z)$; then $\Psi^{*} \in \mathscr{P}$ and $F^{*}(z)=-\Psi(z) / g(z)$ satisfies $\left.F^{*}\right|_{v} ^{r} M-F^{*}=\varphi_{M}^{*}$, for $M \in \Gamma$. Defining $F(z)=F^{*}(z)+\varphi_{0}(z)$, we have

$$
\left.F\right|_{v} ^{r} M-F=\varphi_{M}^{*}+\varphi_{0} \mid M-\varphi_{0}=\varphi_{M}, \quad \text { for } M \in \Gamma \text {. }
$$

The first problem that presents itself is the possibility that $g(z) \equiv 0$; this indeed does happen for certain choices of $\Gamma, k$, and $w$. However, if we simply choose $k$ an even integer and $w(V)=1$ for all $V \in \Gamma$, then $g(z)=\sum_{V \in \mathscr{M}}(c z+d)^{-k}$ is an Eisenstein series of degree $-k$ for $\Gamma$, concerning which it is well known that $\left[15\right.$, p. 278] $\lim _{z \rightarrow i \infty} g(z)=2$. Indeed, $g \in C^{+}(\Gamma,-k, 1)$ but $g \notin C^{0}(\Gamma,-k, 1)[15$, p. 278]. Thus, in particular, $g(z) \not \equiv 0$, and $F$ is meromorphic in $\mathscr{H}$.

Since $g$ has at worst finitely many zeros in $\overline{\mathscr{R}} \cap \mathscr{H}[15$, p. 274], $F$ has at worst finitely many poles in $\overline{\mathscr{R}} \cap \mathscr{H}$. For each $j, 0 \leqq j \leqq t$, consider the function $F_{j}=F-\varphi_{j}$. Then we have

$$
F_{j} \mid Q_{j}-F_{j}=\left(F \mid Q_{j}-F\right)-\left(\varphi_{j} \mid Q_{j}-\varphi_{j}\right)=\varphi_{Q_{j}}-\varphi_{Q_{j}}=0 ;
$$

by a standard argument it follows from this that

and

$$
F_{j}(z)=\left(z-q_{j}\right)^{r} \sum_{m=-\infty}^{\infty} a_{m}(j) \exp \left\{\frac{-2 \pi i\left(m+\kappa_{j}\right)}{\lambda_{j}\left(z-q_{j}\right)}\right\}, \quad 1 \leqq j \leqq t,
$$

$$
F_{0}(z)=\sum_{m=-\infty}^{\infty} a_{m}(0) \exp \left\{\frac{2 \pi i(m+\kappa) z}{\lambda}\right\} .
$$

However since $g \in C^{+}(\Gamma,-k, 1)$ it has at worst a zero of finite order, in the appropriate local variable, at each $q_{j}$. This, together with the fact that $\varphi_{j} \in \mathscr{P}$, implies that $F(z)$ has expansions of the form (2.3) at the parabolic points.

Since $F$ may have poles in $\mathscr{H}$ we need to modify it somewhat to obtain the function $\Phi$ of Theorem 3. It follows from results of Petersson [22] that there exists $f \in\{\Gamma, r, v\}$ which has poles with given principal parts at finitely many points of $\overline{\mathscr{R}} \cap \mathscr{H}$ and is otherwise holomorphic in $\overline{\mathscr{R}}$ with the possible exception of the cusps. Then we form $\Phi=F-f$, where $f \in\{\Gamma, r, v\}$ is chosen to have poles whose principal parts agree with those of $F$ in $\overline{\mathscr{R}} \cap \mathscr{H}$. Since $\left.f\right|_{v} ^{r} M=f$ for $M \in \Gamma$, it is still the case that $\left.\Phi\right|_{v} ^{r} M-\Phi=\varphi_{M}, M \in \Gamma$. At each parabolic cusp $f$ has at worst a pole of finite order; thus $\Phi$ has expansions of the form (2.3) at the parabolic cusps. Finally, $\Phi$ is holomorphic in $\overline{\mathscr{R}} \cap \mathscr{H}$; this and the functional equation $\Phi \mid M-\Phi=\varphi_{M}, M \in \Gamma$, imply that $\Phi$ is holomorphic in $\mathscr{H}$. This completes the proof of Theorem 3 . 
III. Proof of Theorem 1. 1. The case $r \leqq-2$. In this case the result is $\tilde{H}_{r, v}^{1}(\Gamma, \mathscr{P})=\{0\}$. In the proof we need

LEMMA 8. There exist positive constants $K, \rho_{0}, \sigma_{0}$ such that for all $\tau \in \overline{\mathscr{R}} \cap \mathscr{H}$ and $V \in \mathscr{M}$,

where $y=\operatorname{Im}(V \tau)$.

$$
\left|\varphi_{V}(\tau)\right|<K\left(y^{\rho_{0}}+y^{-\sigma_{0}}\right),
$$

Proof. Fix $\tau \in \overline{\mathscr{R}} \cap \mathscr{H}, V \in \mathscr{M}$, and put $z=V \tau$. Write $V=C_{1} \cdots C_{q}$, a product of sections, as before. Then, as in the derivation of (2.14), we have

$$
\left|\varphi_{V}(\tau)\right| \leqq K_{2}^{*}\left(c^{2}+d^{2}\right)^{e+1}\left(|\tau|^{\eta}+t^{-\eta}\right),
$$

where $t=\operatorname{Im} \tau$ and $V=\left(\begin{array}{cc}a & b \\ c & d\end{array}\right)$. By Lemma $4, c^{2}+d^{2} \leqq|c \tau+d|^{2}\left(1+4|\tau|^{2}\right) t^{-2}$. Now,

$$
y=\operatorname{Im} z=\operatorname{Im} V \tau=t|c \tau+d|^{-2},
$$

so that $|c \tau+d|^{2}=t / y$. Hence $c^{2}+d^{2} \leqq(t y)^{-1}\left(1+4|\tau|^{2}\right)$, from which it follows that

$$
\left|\varphi_{V}(\tau)\right| \leqq K_{2}^{*}\left(1+4|\tau|^{2}\right)^{e+1}(t y)^{-e-1}\left(|\tau|^{\eta}+t^{-\eta}\right) .
$$

If $V \in \mathscr{M}, V \neq I$, then $c \neq 0$; hence $|c \tau+d|^{2} \geqq 1$, for all $V \in \mathscr{M}$, since $\tau \in \overline{\mathscr{R}}$ and thus $\tau$ is interior to no isometric circle. If follows that $y=t|c \tau+d|^{-2} \leqq t$. On the other hand, $\tau=V^{-1} z$, so that $t=\operatorname{Im}\left(V^{-1} z\right)=$ $y|-c z+a|^{-2}$. Now,

$$
|-c z+a|^{2}=c^{2} y^{2}+(a-c x)^{2} \geqq c^{2} y^{2}>m_{3}^{2} y^{2},
$$

unless $c=0$; if $c=0$, then $t=y$. Thus $t=y$ or $t<m_{3}^{-2} y^{-1}$, so that in every case, $t<y+m_{3}^{-2} y^{-1}$. Also $\tau \in \overline{\mathscr{R}}$ implies that $|\operatorname{Re} \tau| \leqq \lambda / 2$, hence

$$
|\tau| \leqq t+\lambda / 2<y+m_{3}^{-2} y^{-1}+\lambda / 2 \text {. }
$$

Applying these estimates in (3.1), we find that

$$
\begin{aligned}
&\left|\varphi_{V}(\tau)\right|<K_{2}^{*} \frac{\left\{1+4\left(y+m_{3}^{-2} y^{-1}+\lambda / 2\right)^{2}\right\}^{e+1}}{y^{2 e+2}} \\
& \times\left\{\left(y+m_{3}^{-2} y^{-1}+\lambda / 2\right)^{\eta}+y^{-\eta}\right\},
\end{aligned}
$$

from which the result follows.

Proof of THEOREM 1 FOR $r<-2$. We shall show that if $r \leqq-2$ and $\left\{\varphi_{V}\right\}$ is a parabolic cocycle in $\mathscr{P}$, then there exists $\Phi \in \mathscr{P}$ such that

$$
\left.\Phi\right|_{v} ^{r} M-\Phi=\varphi_{M}, \quad M \in \Gamma .
$$

As in the proof of Theorem 3 we may, without loss of generality, assume that $\varphi_{S}=0$, since $\varphi_{0} \in \mathscr{P}$. Let $\Phi$ be the function whose existence is 
guaranteed by Theorem 3. Since $r \leqq-2$ there exists $G \in\{\Gamma, r, v\}$ such that $G$ is holomorphic in $\mathscr{H}$ and $G$ has a principal part at each $q_{j}$ which agrees precisely with the principal part at $q_{j}$ of the expansion (2.3) of $\Phi$. (If $r<-2, G$ can be constructed as a linear combination of Poincaré series, whose absolute convergence is guaranteed in this case [15, pp. 272280]. If $r=-2$ the much more difficult construction has been carried out by Petersson [20], [21].) If we put $\Phi^{*}=\Phi-G$, then

(i) $\Phi^{*}{ }_{v}^{r} M-\Phi^{*}=\varphi_{M}, M \in \Gamma$,

(ii) $\Phi^{*}$ is holomorphic in $\mathscr{H}$,

(iii) $\Phi^{*}$ has an expansion (2.3) at each $q_{j}$ in which no negative power of the local parameter appears, $0 \leqq j \leqq t$.

Condition (iii) implies that there are positive constants $K_{\mathscr{R}}, \rho, \sigma$ such that

$$
\left|\Phi^{*}(z)\right|<K_{\mathscr{R}}\left(|y|^{\rho}+y^{-\sigma}\right), \text { for all } z \in \overline{\mathscr{R}} \cap \mathscr{H} .
$$

Put $f(z)=y^{-r / 2}\left|\Phi^{*}(z)\right|, y=\operatorname{Im} z>0$. Then for $V=\left(\begin{array}{cc}* & * \\ c & a\end{array}\right) \in \Gamma$,

$$
\begin{aligned}
f(V z) & =y^{-r / 2}|c z+d|^{r}\left|\Phi^{*}(V z)\right| \\
& =y^{-r / 2}|c z+d|^{r}\left|(c z+d)^{-r} \Phi^{*}(z)+(c z+d)^{-r} \varphi_{V}(z)\right| \\
& =y^{-r / 2}\left|\Phi^{*}(z)+\varphi_{V}(z)\right| \leqq y^{-r / 2}\left|\Phi^{*}(z)\right|+y^{-r / 2}\left|\varphi_{V}(z)\right| \\
& =f(z)+y^{-r / 2}\left|\varphi_{V}(z)\right| .
\end{aligned}
$$

Let $\mathscr{S}=\bigcup_{V \in \mathscr{M}} V(\overline{\mathscr{R}}) \cap \mathscr{H}$. Suppose we can prove that

$$
\left|\Phi^{*}(z)\right|<K\left(y^{\alpha}+y^{-\beta}\right), \text { for } z \in \mathscr{S},
$$

with $\alpha, \beta, K$ positive constants independent of $z$. From the definition of fundamental region and the choice of $\mathscr{M}$ it follows that given $z \in \mathscr{H}$, $S^{m} z \in \mathscr{S}$ for some rational integer $m$. Now,

$$
\left|\Phi^{*}\left(S^{m} z\right)\right|=\left|\left(\Phi^{*} \mid S^{m}\right)\right|=\left|\Phi^{*}(z)\right|,
$$

since $\varphi_{S}=0$ (hence $\varphi_{S^{m}}=0$ ); also $\operatorname{Im}\left(S^{m} z\right)=\operatorname{Im} z=y$. Then (2.17) implies that, for $z \in \mathscr{H}$,

$$
\left|\Phi^{*}(z)\right|=\left|\Phi^{*}\left(S^{m} z\right)\right|<K\left(y^{\alpha}+y^{-\beta}\right),
$$

so that, in fact, $\Phi^{*} \in \mathscr{P}$.

It is sufficient, therefore, to prove (3.3). Suppose $z \in \mathscr{S}$; then there exist $V \in \mathscr{M}$ and $\tau \in \overline{\mathscr{R}} \cap \mathscr{H}$ such that $z=V \tau$. Let $y=\operatorname{Im} z$. By the inequality (3.2),

$$
\begin{aligned}
\Phi^{*}(z) & =y^{r / 2} f(z)=y^{r / 2} f(V \tau) \\
& \leqq y^{r / 2}\left\{f(\tau)+t^{-r / 2}\left|\varphi_{V}(\tau)\right|\right\} \\
& =y^{r / 2} t^{-r / 2}\left\{\left|\Phi^{*}(\tau)\right|+\left|\varphi_{V}(\tau)\right|\right\} .
\end{aligned}
$$


Since $\tau \in \overline{\mathscr{R}} \cap \mathscr{H}, \Phi^{*}(\tau)<K_{\mathscr{R}}\left(t^{\rho}+t^{-\sigma}\right)$, while, by Lemma $8,\left|\varphi_{V}(\tau)\right|<$ $K_{1}\left(y^{\rho_{0}}+y^{-\sigma_{0}}\right)$. From (3.4) we conclude that

$$
\Phi^{*}(z) \leqq y^{r / 2} t^{-r / 2}\left\{K_{\mathscr{R}}\left(t^{\rho}+t^{-\sigma}\right)+K_{1}\left(y^{\rho_{0}}+y^{-\sigma_{0}}\right)\right\} .
$$

In the proof of Lemma 8 we showed that $y \leqq t \leqq\left(m_{4} y\right)^{-1}+y$. Since $-r / 2>0$,

$$
\Phi^{*}(z) \leqq y^{r / 2}\left(\frac{1}{m_{4} y}+y\right)^{-r / 2}\left\{K_{\mathscr{R}}\left(\frac{1}{m_{4} y}+y\right)^{\rho}+y^{-\sigma}+K_{1}\left(y^{\rho_{0}}+y^{-\sigma_{0}}\right)\right\},
$$

from which (3.3) follows and the proof is complete for $r \leqq-2$.

REMARK. It is important to notice that the above line of proof depends on the fact that $r \leqq-2$ only for the existence of $G \in\{\Gamma, r, v\}$ with preassigned singularities at the cusps. The existence of such $G$ is a kind of Mittag-Leffler theorem for automorphic forms which does not hold in general for $r>-2$. For the case $r>0$ we substitute recent results of Douglas Niebur on automorphic integrals of arbitrary positive degree [18]. For $r=0$ we again apply Petersson's construction of automorphic forms of degree -2 [20], [21].

2. The case $r>0$. For any real $r$ the previously-quoted results of Petersson [22] imply that there exists $f \in\{\Gamma, r, v\}$ which has poles of prescribed principal parts at each of the cusps $q_{1}, \cdots, q_{t}$ and is holomorphic in $\mathscr{H}$. If $r \geqq 0$ one has no control over the principal part at the cusp $q_{0}=\infty$; this principal part is, indeed, largely (not completely) determined by the assignment of the principal parts at $q_{1}, \cdots, q_{t}$. With fixed $r \geqq 0$ and given parabolic cocycle $\left\{\varphi_{V}\right\}$ in $\mathscr{P}$, let $\Phi$ be the function of Theorem 3 and let $f \in\{\Gamma, r, v\}$ be holomorphic in $\mathscr{H}$ with principal parts as given by $(2.3)$ for $1 \leqq j \leqq t$. Then

$$
\Phi *(z)=\Phi(z)-f(z)
$$

satisfies all of the conclusions of Theorem 3 and, in addition, is holomorphic at the finite cusps $q_{1}, \cdots, q_{t}$.

With $r>0$ and $v$ an associated multiplier system for the $H$-group $\Gamma$, the results of Niebur that we shall require may be stated as follows [18]:

THEOREM $\mathrm{N}_{1}$. Let $m_{0}$ be a nonnegative integer and $a_{-1}, \cdots, a_{-m_{0}}$ complex numbers. Then there exists $F$, an automorphic integeral of degree $r$ with respect to $\Gamma$, which is holomorphic in $\mathscr{H}$ and at the finite cusps $q_{1}, \cdots, q_{t}$ and which has an expansion (2.3) with principal part

$$
a_{-m_{0}} \exp \left\{2 \pi i\left(-m_{0}+\kappa\right) z / \lambda\right\}+\cdots+a_{-1} \exp \{2 \pi i(-1+\kappa) z / \lambda\}
$$


at $q_{0}=\infty$. The function $F$ has the transformation properties

$$
\begin{aligned}
\left.F\right|_{v} ^{r} V & =F, \quad V \in \Gamma_{\infty}, \\
{\left[\left.F\right|_{v} ^{r} V-F\right]^{-} } & =\int_{V_{\infty}^{-1}}^{i \infty} G(\tau)(\tau-\bar{z})^{r} d \tau, \quad V \in \Gamma-\Gamma_{\infty},
\end{aligned}
$$

where $G \in C^{0}(\Gamma,-r-2, \bar{v})$ is determined by $a_{-m_{0}}, \cdots, a_{-1}$. Here $[h(z)]^{-}$ denotes $\overline{h(z)}$, the complex conjugate of $h(z)$, and the path of integration is a vertical line. Furthermore, $\{F \mid V-F\}$ is a parabolic cocycle in $\mathscr{P}$.

TheOREM $\mathrm{N}_{2}$. Given $G \in C^{0}(\Gamma,-r-2, \bar{v})$ there exists an automorphic integral $F$ satisfying (3.7) such that $F$ is holomorphic in $\mathscr{H}$ and at $q_{1}, \cdots, q_{t}$.

REMARK. Theorem $\mathrm{N}_{2}$, a converse of Theorem $\mathrm{N}_{1}$, is analogous to our Theorem 3 as it allows one to prescribe the period functions of an automorphic integral. As in Theorem 3, with the prescription of the period functions one loses the freedom to prescribe the principal part at $\infty$, which is, indeed, largely determined by the given $G$.

ThEOREM $\mathrm{N}_{3}$. If there exists $\tilde{F} \in\{\Gamma, r, v\}$ which is holomorphic in $\mathscr{H}$ and at $q_{1}, \cdots, q_{t}$, and which has principal part (3.6) at $q_{0}=\infty$, then the function $F$ of Theorem $\mathrm{N}_{1}$ is in $\{\Gamma, r, v\}$ and, in fact, $F=\widetilde{F}$. In this case the cusp form $\boldsymbol{G}$ of (3.7) is $\equiv 0$.

Proof of Theorem 1 for $\grave{r}>0$. Suppose $G \in C^{0}(\Gamma,-r, \bar{v})$. Then put $\alpha(G)=\left\langle g_{V}\right\rangle$, where

$$
\overline{g_{V}(z)}=\int_{V_{\infty}^{-1}}^{i \infty} G(\tau)(\tau-\bar{z})^{r} d \tau, \quad V \in \Gamma,
$$

and $\left\langle g_{V}\right\rangle$ denotes the cohomology class in $H_{r, v}^{1}(\Gamma, \mathscr{P})$ determined by $\left\{g_{V}\right\}$ which, by Theorem $\mathrm{N}_{2}$, is a parabolic cocycle in $\mathscr{P}$. If $V \in \Gamma_{\infty}$ the integral in (3.8) is to be interpreted as 0 ; for $V \in \Gamma-\Gamma_{\infty}$ the path of integration is a vertical line. The mapping $\alpha$ is obviously linear from $C^{0}(\Gamma,-r-2, \bar{v})$ into $\widetilde{H}_{r, v}^{1}(\Gamma, \mathscr{P})$. We must show that $\alpha$ is $1-1$ and onto.

To show that $\alpha$ is onto suppose $\left\langle\varphi_{V}\right\rangle$ is an element of $\tilde{H}_{r, v}^{1}(\Gamma, \mathscr{P})$. Let $\Phi(z)$ be the function of Theorem 3 with cocycle of period functions $\left\{\varphi_{V}\right\}$ and let $\Phi^{*}(z)$ be given by (3.5). Then $\Phi^{*}$ has the prescribed cocycle of period functions $\left\{\varphi_{V}\right\}$ and it is holomorphic in $\mathscr{H}$ and at the finite parabolic cusps $q_{1}, \cdots, q_{t}$ as well. Apply Theorem $\mathrm{N}_{1}$ to obtain the existence of an automorphic integral $F$ such that $\Phi=\Phi^{*}-F$ is again an automorphic integral of degree $r$ with parabolic period functions in $\mathscr{P}$ and such that $\Phi$ is holomorphic in $\mathscr{H}$ and at all of the parabolic cusps (including $q_{0}=\infty$ ). The argument used in §IIII.1 to conclude the proof of Theorem 1 for $r \leqq-2$ now shows that $\Phi \in \mathscr{P}$, from which it follows that 
$\left\langle\varphi_{V}\right\rangle=\left\langle g_{V}\right\rangle$, with $g_{V}$ determined by (3.8) from $G \in C^{0}(\Gamma,-r-2, \bar{v})$, which, in turn, is determined from $F$ by Theorem $N_{1}$. Then $\alpha(G)=\left\langle\varphi_{V}\right\rangle$ and $\alpha$ is onto.

To show that $\alpha$ is $1-1$ suppose that $\alpha(G)=\left\langle g_{V}\right\rangle=0$ in $\tilde{H}_{r, v}^{1}(\Gamma, \mathscr{P})$ for some $G \in C^{0}(\Gamma,-r-2, \bar{v})$. This means that there exists $\hat{F} \in \mathscr{P}$ such that $g_{V}=\left.\hat{F}\right|_{v} ^{r} V-\hat{F}, V \in \Gamma$. On the other hand, Theorem $\mathrm{N}_{2}$ implies that there exists an automorphic integral $F$ holomorphic in $\mathscr{H}$ and at $q_{1}, \cdots, q_{t}$ such that $g_{V}=\left.F\right|_{v} ^{r} V-F, V \in \Gamma$. It follows that $\tilde{F}=F-\hat{F} \in\{\Gamma, r, v\}$ and $\tilde{F}$ is holormophic in $\mathscr{H}$ and at $q_{1}, \cdots, q_{t}$. At $\infty \tilde{F}$ has the same principal part as does $F$. Theorem $\mathrm{N}_{3}$ then implies that $F=\tilde{F}$ and $G \equiv 0$. Thus $\alpha$ is $1-1$ and the proof of Theorem 1 is complete for the case $r>0$.

3. The case $r=0$. If $r=0$ the results of Niebur are not applicable and we apply instead results of Petersson which are utilized in [6] to prove Theorem A for the case $r=0$.

Since $v(S)=e^{2 \pi i \kappa}, 0 \leqq \kappa<1$, it follows that $\bar{v}(S)=e^{2 \pi i \kappa^{\prime}}$, with

If $v$ is an integer define

$$
\begin{aligned}
\kappa^{\prime} & =0 & & \text { if } \kappa=0, \\
& =1-\kappa & & \text { if } \kappa>0 .
\end{aligned}
$$

$$
\begin{aligned}
\nu^{\prime} & =-v & & \text { if } \kappa=0 \\
& =-1-v & & \text { if } \kappa>0
\end{aligned}
$$

In [20], [21] Petersson has carried out a construction of automorphic forms of degree -2 with arbitrary multiplier system $v$ on $H$-groups $\Gamma$. He produces functions $g_{v}(z, v)$ holomorphic in $\mathscr{H}$ such that

(i) $g_{v}(z, v) \in\{\Gamma,-2, v\}$,

(ii) $g_{v}(z, v)$ is zero at $q_{1}, \cdots, q_{t}$. At $q_{0}=\infty$ it has an expansion of the form

$$
q_{v}(z, v)=2 \exp \left\{\frac{2 \pi i(v+\kappa) z}{\lambda}\right\}+2 \sum_{m+\kappa>0} a_{m}(v, v) \exp \left\{\frac{2 \pi i(m+\kappa) z}{\lambda}\right\} .
$$

In particular if $v+\kappa>0$ then $g_{v}(z, v) \in C^{0}(\Gamma,-2, v)$.

Petersson Gap Theorem [19, p. 207 and p. 211, Theorem 9 $\alpha$ ]. Let $s$ be the complex dimension of the vector space $C^{0}(\Gamma,-2, \bar{v})$. Then there are precisely $s$ integers $w_{i}, 0<w_{1}<\cdots<w_{s}$, such that there does not exist a nonconstant element of $\{\Gamma, 0, v\}$ having as its only singularity in $\overline{\mathscr{R}} \cap \mathscr{H}$ a pole at $q_{0}=\infty$ of order $w_{i}-\kappa, 1 \leqq i \leqq s$, in the local uniformizing variable at $\infty$. Furthermore the functions $g_{\left(-w_{i}\right)},(z, \bar{v}), 1 \leqq i \leqq s$, form a basis for $C^{0}(\Gamma,-2, \bar{v})$.

Proof of Theorem 1 for $r=0$. Suppose $G \in C^{0}(\Gamma,-2, \bar{v})$. Then $G=\sum_{i=1}^{s} b_{i} g_{\left(-w_{i}\right)}(z, \bar{v})$. Let $G^{*}=\sum_{i=1}^{s} \bar{b}_{i} g_{-w_{i}}(z, v) \in\{\Gamma,-2, v\}$. Note that 
$G^{*}$ is holomorphic in $\mathscr{H}$, zero at $q_{1}, \cdots, q_{t}$, and has an expansion at $q_{0}=\infty$ with principal part $2 \sum_{i=1}^{s} b_{i} \exp \left\{2 \pi i\left(-w_{i}+\kappa\right) z / \lambda\right\}$. Let $F$ be holomorphic in $\mathscr{H}$ and such that $d F(z) / d z=G^{*}(z)$, so normalized that $F(z+\lambda)=e^{2 \pi i \kappa} F(z)$. Then $F$ is an automorphic integral of degree 0 with a parabolic cocycle of period functions. In addition $F$ is holomorphic at $q_{1}, \cdots, q_{t}$ and has principal part at $q_{0}=\infty$

$$
2 \sum_{i=1}^{s} \bar{b}_{i}\left\{\frac{2 \pi i\left(-w_{i}+\kappa\right)}{\lambda}\right\}^{-1} \exp \left\{\frac{2 \pi i\left(-w_{i}+\kappa\right) z}{\lambda}\right\} .
$$

Furthermore, in this case $\left.F\right|_{0} ^{0} V-F=c_{V}, V \in \Gamma$, where $c_{V}$ is a complex number. Now we define $\alpha(G)=\left\langle c_{V}\right\rangle$, where $\left\langle c_{V}\right\rangle$ denotes the cohomology class in $H_{0, v}^{1}(\Gamma, \mathscr{P})$ of the cocycle $\left\{c_{V}\right\}$. Clearly $\alpha$ is a linear mapping of $C^{0}(\Gamma,-2, \bar{v})$ into $\tilde{H}_{0, v}^{1}(\Gamma, \mathscr{P})$. As before we must show that $\alpha$ is 1-1 and onto.

Suppose $\left\langle\varphi_{V}\right\rangle \in \tilde{H}_{\mathbf{0}, v}^{1}(\Gamma, \mathscr{P})$; let $\Phi$ be the corresponding function of Theorem 3 and let $\Phi^{*}$ be defined by (3.5) once again. Then $\Phi^{*}$ is holomorphic in $\mathscr{H}$ and at the finite parabolic cusps $q_{1}, \cdots, q_{t}$ and has $\left\{\varphi_{V}\right\}$ as its cocycle of period functions. Petersson's construction yields the existence of $G \in C^{0}(\Gamma,-2, \bar{v})$ such that the automorphic integral $F$, the anti-derivative of the corresponding $G^{*} \in\{\Gamma,-2, v\}$, has the property that $\hat{\Phi}=\Phi-F$ is holomorphic in $\overline{\mathscr{R}}$. Once again, the earlier argument implies that $\Phi \in \mathscr{P}$. From this it follows that $\left\langle\varphi_{V}\right\rangle=\left\langle c_{V}\right\rangle$, where $c_{V}=F \mid{ }_{v}^{0} V-F, V \in \Gamma$, and therefore $\alpha(G)=\left\langle c_{V}\right\rangle=\left\langle\varphi_{V}\right\rangle$. Thus $\alpha$ is onto $\tilde{H}_{0, v}^{1}(\Gamma, \mathscr{P})$.

Now suppose that $\alpha(G)=\left\langle c_{V}\right\rangle=0$ in $H_{0, v}^{1}(\Gamma, \mathscr{P})$ for some $G \in C^{0}(\Gamma$, $-2, \bar{v})$. Then there exists $\hat{F} \in \mathscr{P}$ such that $c_{V}=\hat{F} \mid{ }_{v}^{0} V-\hat{F}, V \in \Gamma$. On the other hand, if $F$ is the anti-derivative of the $G^{*} \in\{\Gamma,-2, v\}$ corresponding to $G$ (as above), then also $c_{V}=F{ }_{v}^{0} V-F, V \in \Gamma$, so that

$$
\widetilde{F}=F-\hat{F} \in\{\Gamma, 0, v\} .
$$

Since $\hat{F} \in \mathscr{P}, \widetilde{F}$ is holomorphic in $\bar{R}$, except possibly at $q_{0}=\infty$ where it has the same principal part as does $F$, given by (3.9). The Petersson gap theorem then implies that $F$ is constant, that is, that $b_{i}=0$ for $1 \leqq i \leqq s$. From this it follows that $G \equiv 0$, so that $\alpha$ is $1-1$ and the proof of Theorem 1 is complete.

ReMARK. For any $r$ it is obvious that with given $\Phi \in \mathscr{P},\left\{\left.\Phi\right|_{v} ^{r} V-\Phi\right\}$ is a cocycle in $\mathscr{P}$. (Of course it is a coboundary in $\mathscr{P}$.) For $r \leqq-2$ Theorems 1 and 2 have the interpretation that these easily available cocycles are, in fact, the only cocycles in $\mathscr{P}$. For $r>0$ Theorem $\mathrm{N}_{1}$ implies the existence of cocycles in $\mathscr{P}$ which are not obviously coboundaries in $\mathscr{P}$. Theorems 1 and 2 in this case show that every cocycle in $\mathscr{P}$ is one of the cocycles of Theorem $\mathrm{N}_{1}$ plus a coboundary in $\mathscr{P}$. 
IV. Proof of Theorem 2. 1. Deduction of Theorem 2 from Taylor's proposition. Theorem 2 follows from the fact that, for any real $r$,

$$
H_{r, v}^{1}(\Gamma, \mathscr{P})=\tilde{H}_{r, v}^{1}(\Gamma, \mathscr{P}),
$$

which in turn is a consequence of the following result concerning $\mathscr{P}$ due to B. A. Taylor (private communication).

Proposition 9. Suppose $g \in \mathscr{P}$ and $\varepsilon$ is a complex number with $|\varepsilon|=1$. Then there exists $f \in \mathscr{P}$ such that

$$
\bar{\varepsilon} f(z+1)-f(z)=g(z), \quad z \in \mathscr{H} .
$$

(Compare this result with [5, Lemma 4]).

We postpone the proof of Proposition 9 for the time being and use it to derive (4.1). To prove (4.1) it is sufficient to show that, given a parabolic transformation $Q \in \Gamma$ and $\varphi \in \mathscr{P}$, there exists $\rho \in \mathscr{P}$ such that

$$
\left.\rho\right|_{v} ^{r} Q-\rho=\varphi \text {. }
$$

There are two cases to consider.

Suppose first that $Q$ is a translation, that is, $Q=\left(\begin{array}{ll}1 & \lambda \\ 0 & 1\end{array}\right), \lambda>0$. Put $g(z)=$ $\varphi(\lambda z)$; then $g \in \mathscr{P}$ and we may apply Proposition 9 to conclude that there exists $f \in \mathscr{P}$ such that

$$
\bar{v}(Q) f(z+1)-f(z)=g(z), \quad z \in \mathscr{H} .
$$

Put $\rho(z)=f(z / \lambda)$; then $\rho \in \mathscr{P}$ and

$$
\begin{aligned}
\rho{ }_{v}^{r} Q-\rho & =\bar{v}(Q) \rho(z+\lambda)-\rho(z)=\bar{v}(Q) f((z+\lambda) / \lambda)-f(z / \lambda) \\
& =\bar{v}(Q) f(z / \lambda+1)-f(z / \lambda)=g(z / \lambda)=\varphi(z),
\end{aligned}
$$

for any $z \in \mathscr{H}$. Thus (4.3) has a solution if $Q$ is a translation.

If $Q \in \Gamma$ is parabolic, but not a translation, then $Q$ can be written in the form $Q=A^{-1}\left(\begin{array}{ll}1 & \lambda \\ 0 & 1\end{array}\right) A, \lambda>0$, where $A$ is a real matrix of determinant 1 such that $A q=\infty$. Here $q$ is the parabolic point fixed by $Q$. By the previous case, given $\varepsilon,|\varepsilon|=1$, and $g \in \mathscr{P}$, there exists $f \in \mathscr{P}$ such that

$$
\bar{\varepsilon} f(z+\lambda)-f(z)=g(z), \quad z \in \mathscr{H} \text {. }
$$

Suppose $Q=\left(\begin{array}{cc}* & * \\ c & d\end{array}\right), A=\left(\begin{array}{cc}* & * \\ \gamma & \delta\end{array}\right)$ and put

$$
\varepsilon=v(Q)(c z+d)^{-r}(\gamma Q z+\delta)^{-r}(\gamma z+\delta)^{r} .
$$

To show that $|\varepsilon|=1$ it is sufficient to prove

$$
(c z+d)(\gamma Q z+\delta)=\gamma z+\delta .
$$

But a simple calculation shows that $(c z+d)(\gamma Q z+\delta)$ is the denominator of $A Q z$. Since $A Q z=\left(\begin{array}{ll}1 & \lambda \\ 0 & 1\end{array}\right) A z$ and $\left(\begin{array}{ll}1 & \lambda \\ 0 & 1\end{array}\right) A z$ has the same denominator as 
$A z$, namely $\gamma z+\delta$, the equality (4.6) holds and $|\varepsilon|=1$. Since $|\varepsilon|=1$ and $\varepsilon$ is holomorphic (as a function of $z$ ) in $\mathscr{H}$, it follows that $\varepsilon$ is constant and (4.4) may be applied.

Suppose $g \in \mathscr{P}, \varepsilon$ is given by (4.5), and $f \in \mathscr{P}$ is a solution of (4.4). Put $\rho(z)=(\gamma z+\delta)^{r} f(A z)$; then $\rho \in \mathscr{P}$ and

$$
\begin{aligned}
\left.\rho\right|_{v} ^{r} Q-\rho & =\bar{v}(Q)(c z+d)^{r} \rho(Q z)-\rho(z) \\
& =\bar{v}(Q)(c z+d)^{r}(\gamma Q z+\delta)^{r} f(A Q z)-\rho(z) \\
& =\bar{\varepsilon}(\gamma z+\delta)^{r} f(A z+\lambda)-(\gamma z+\delta)^{r} f(A z) \\
& =(\gamma z+\delta)^{r}\{\bar{\varepsilon} f(A z+\lambda)-f(A z)\} \\
& =(\gamma z+\delta)^{r} g(A z),
\end{aligned}
$$

by (4.4) with $z$ replaced by $A z$. We now put

$$
g(z)=\left(\gamma A^{-1} z+\delta\right)^{-r} \varphi\left(A^{-1} z\right),
$$

so that $(\gamma z+\delta)^{r} g(A z)=\varphi(z)$, the given function in $\mathscr{P}$. With $\varphi \in \mathscr{P}, g$ is likewise in $\mathscr{P}$. Thus (4.3) has a solution $\rho \in \mathscr{P}$ for any parabolic $Q \in \Gamma$ and (4.1) follows.

2. Proof of Taylor's result. A formal solution to the functional equation (4.2), with given $g$, is provided by the function

$$
f(z)=-\sum_{n=0}^{\infty} \bar{\varepsilon}^{n} g(z+n) .
$$

Indeed, if $g \in \mathscr{P}$ vanishes at $\infty$ with sufficient rapidity (say $g(z)=\mathcal{O}\left(|z|^{-2}\right)$ as $|z| \rightarrow \infty$ ), then (4.7) converges absolutely in $\mathscr{H}$ and uniformly on compact subsets of $\mathscr{H}$. In this case it is easy to verify that $f \in \mathscr{P}$. Though, in general, the series (4.7) does not converge, it nevertheless remains the key to the solution of (4.2).

In order to overcome the convergence difficulties we first observe that it is sufficient to solve (4.2) for the $k$ th derivative of $g$ or a $k$-fold indefinite integral of $g$. This follows since $\mathscr{P}$ is stable under differentiation and integration and since (4.2) is readily solvable in $\mathscr{P}$ if $g$ is a polynomial. (Indeed if $g \in P_{k},(4.2)$ is solvable with $f \in P_{k+1}$.) We also make the easily verified observation that the condition (1.11) for $g \in \mathscr{P}$ may be replaced by $|g(z)|<K(1+|z|)^{\alpha} y^{-\beta}, y=\operatorname{Im} z$, with $K, \alpha, \beta$ all positive, or by

$$
\begin{aligned}
|g(z)| & <K(1+|z|)^{\alpha}, & & y \geqq 1, \\
& <K(1+|z|)^{\alpha} y^{-\beta}, & & 0<y<1,
\end{aligned}
$$

again with $K, \alpha, \beta>0$. 
LEMMA 10. Suppose $g \in \mathscr{P}$ satisfies (4.8) and $h$ is any indefinite integral of $g(d h / d z=g(z), z \in \mathscr{H})$.

(a) If $\beta>1$ then $h$ satisfies (4.8) with $\alpha$ replaced by $\alpha+1$ and $\beta$ replaced by $\beta-1$. (The constant $K$ must be replaced as well.)

(b) If $0<\beta<1$ then $h$ is continuous in $\mathscr{H}$ (i.e. in $y \geqq 0$ ), and there satisfies

$$
|h(z)|<K^{\prime}(1+|z|)^{\alpha+1} .
$$

The proof of (a) employs a straightforward use of (4.8) and Cauchy's theorem. The proof of (b) is more complicated since the proof that $\lim _{z \rightarrow x} h(z)=h(x), z=x+i y, y>0$, exists is somewhat delicate. It involves a careful (though not deep) analysis and employs, once again, Cauchy's theorem. The details will be omitted.

Lemma 10 shows that if $g \in \mathscr{P}$ then some $k$-fold indefinite integral of $g$ is continuous in $\overline{\mathscr{H}}$ and satisfies an inequality of the form (4.9). (If $\beta$ is not an integer take $k=[\beta]+1$. If $\beta$ is an integer replace $\beta$ by $\beta+\frac{1}{2}$; then we may choose $k=\beta+1$. Thus in either case $k=[\beta]+1$ will do, where $[\beta]$ is the largest integer $\leqq \beta$.) This fact, together with our previous observation, implies that it is sufficient to prove Theorem 9 in the case when $g \in \mathscr{P}$ is continuous in $\overline{\mathscr{H}}$ and satisfies

$$
|g(z)|<K(1+|z|)^{\alpha}, \quad y=\operatorname{Im} z \geqq 0,
$$

with $K, \alpha>0$. On the other hand, given such $g$, if $k$ is an integer $>\alpha+1$ then

$$
g^{(k-1)}(z)=\frac{(k-1) !}{2 \pi i} \int_{-\infty}^{\infty} \frac{g(u)}{(u-z)^{k}} d u, \quad z \in \mathscr{H} .
$$

In order to prove (4.11) let $C_{t}, t>0$, be the path in $\overline{\mathscr{H}}$ consisting of the interval $[-t, t]$ followed by the semicircle in $\mathscr{H}$ connecting $t$ to $-t$. The Cauchy Integral Formula shows that

$$
g^{(k-1)}(z)=\frac{(k-1) !}{2 \pi i} \int_{C_{t}} \frac{g(u)}{(u-z)^{k}} d u,
$$

for any $z$ inside $C_{t}$. Letting $t \rightarrow+\infty$ yields (4.11). As we have already observed, it is sufficient to solve (4.2) in $\mathscr{P}$ for $g^{(k-1)}$. Thus we consider

$$
\hat{g}(z)=\int_{-\infty}^{\infty} \frac{g(u)}{(u-z)^{k}} d u
$$

where $g$ satisfies (4.10) and $k$ is an integer $>\alpha+1$. 
LEMMA 11. Write $\hat{g}(z)=\hat{g}_{1}(z)+\hat{g}_{2}(z)$, where

$$
\hat{g}_{1}(z)=\int_{0}^{\infty} \frac{g(u)}{(u-z)^{k}} d u, \quad \hat{g}_{2}(z)=\int_{-\infty}^{0} \frac{g(u)}{(u-z)^{k}} d u .
$$

Then $\hat{g}_{1}, \hat{g}_{2} \in \mathscr{P}$ and for $z \in \mathscr{H}$

$$
\begin{aligned}
\left|\hat{g}_{1}(z)\right| & <K^{\prime}\left(|z|^{1-k}+|z|^{\alpha+1-k}\right), & & x=\operatorname{Re} z \leqq 0, \\
& <K^{\prime \prime}(1+|z|)^{\alpha+1} y^{-k}, & & x=\operatorname{Re} z>0 .
\end{aligned}
$$

The inequality (4.11) holds for $\hat{g}_{2}(z)$, with the conditions on $x$ reversed.

REMARK. (4.11) implies that $\hat{g}_{1} \in \mathscr{P}$.

Once again we omit the details of the proof, which involves estimating the integral representations of $\hat{g}_{1}, \hat{g}_{2}$ by utilizing (4.10).

If we now choose $k$ so large that $k-\alpha-1>1$ then, of course, $k-1>1+\alpha$ and by (4.11) the series

$$
f_{1}(z)=\sum_{n=1}^{\infty} \varepsilon^{n} \hat{g}_{1}(z-n), \quad f_{2}(z)=-\sum_{n=0}^{\infty} \bar{\varepsilon}^{n} \hat{g}_{2}(z+n)
$$

converge absolutely for $z \in \mathscr{H}$ and uniformly on compact subsets of $\mathscr{H}$. Furthermore, an easy calculation shows that $f_{1}, f_{2} \in \mathscr{P}$. Finally, absolute convergence implies that

and

$$
\bar{\varepsilon} f_{1}(z+1)-f_{1}(z)=\hat{g}_{1}(z)
$$

$$
\bar{\varepsilon} f_{2}(z+1)-f_{2}(z)=\hat{g}_{2}(z)
$$

Thus if $f(z)=f_{1}(z)+f_{2}(z)$, then $f \in \mathscr{P}$ and

$$
\bar{\varepsilon} f(z+1)-f(z)=\hat{g}_{1}(z)+\hat{g}_{2}(z)=g(z) .
$$

This completes the proof.

V. Concluding remarks. 1. Theorems 1 and 2 should be compared with Theorem 3 of [5], which, in our notation, may be stated:

$$
C^{0}(\Gamma,-r-2, \bar{v}) \cong H_{r, v}^{1}\left(\Gamma, \mathscr{A}^{0}\right),
$$

where $r \geqq 0$ is an integer, $v$ consists of roots of unity, and $\mathscr{A}^{0}$ is the space of all functions holomorphic in $\mathscr{H}$ which grow more slowly than any exponential at all of the parabolic cusps of $\Gamma$. (See [5, pp. 48-49] for the precise definition of $\mathscr{A}^{0}$.) With $r$ an even integer $(r \neq 0,-2)$ and $v \equiv 1$, Kra [11, Theorems 3 and 6] has shown that

$$
C^{0}(\Gamma,-r-2, \bar{v}) \cong H_{r, v}^{1}(\Gamma, \mathscr{A})
$$


if $\Gamma$ is a finitely generated Fuchsian group of the first kind without parabolic cusps (i.e. $\mathscr{H} / \Gamma$ is compact) and $\mathscr{A}$ is the space of all functions holomorphic in $\mathscr{H}$. Kra's formulation of (5.2) is actually more general than that given here, since he deals with Kleinian groups. Kra has also shown that [11, Theorem 5]

$$
H_{r, v}^{1}(\Gamma, \mathscr{A})=0
$$

for any even integer $r$ and $v \equiv 1$ if $\Gamma$ is a Fuchsian group such that $\mathscr{H} / \Gamma$ is open. This includes the case of $H$-groups $\Gamma$, Again, Kra's formulation is in terms of Kleinian groups $\Gamma$.

2. Comparison of Theorem A, Theorems 1 and 2, (5.1), and (5.3) suggests that $H_{r, v}^{1}(\Gamma, \mathscr{F})$ (with fixed $r \geqq 0, v, \Gamma$ ) gets smaller as $\mathscr{F}$ gets larger. It would be interesting to determine the "largest" $\mathscr{F}$ (if such exists), $\mathscr{P} \subset \mathscr{F} \subset \mathscr{A}$, with the property that $H_{r, v}^{1}(\Gamma, \mathscr{F}) \neq\{0\}$, or, alternatively, such that $H_{r, v}^{1}(\Gamma, \mathscr{F}) \cong C^{0}(\Gamma,-r-2, \bar{v})$. A related question is the determination of the "smallest" $\mathscr{F}, \mathscr{P} \subset \mathscr{F} \subset \mathscr{A}$ such that $H_{r, v}^{1}(\Gamma, \mathscr{F})=\{0\}, r \geqq 0$. In more general terms, it might be of value to determine the behavior of $H_{r, v}^{1}(\Gamma, \mathscr{F})$ as $\mathscr{F}$ varies.

The proof of Theorem 2 shows that, in fact, $H_{r, v}^{1}(\Gamma, \mathscr{P})=\tilde{H}_{r, v}^{1}(\Gamma, \mathscr{P})$ for any group $\Gamma$ of real linear fractional transformations, whether it is finitely generated or not, whether it is discrete or not. We are led to ask whether an analogue of Theorem 1 is true for Fuchsian groups in general, with a suitable replacement for $C^{0}(\Gamma,-r-2, \bar{v})$, of course. Another question that presents itself naturally is whether Theorem $\mathrm{A}$ has an analogue when $r>0$ but $r$ is not an integer. This involves identifying a space of functions to replace $P_{r}$, the polynomials of degree $\leqq r$. Another difficulty in this connection is that, with $r$ not an integer and $\bar{v}$ a multiplier system of degree $-r-2, v$ is not a multiplier system of degree $-r-2$. Thus one must replace $C^{+}(\Gamma,-r-2, v)$ as well.

Finally, I conjecture that Theorem 1 is true in the range $-2<r<0$.

\section{REFERENCES}

1. G. Bol, Invarianten linearer Differentialgleichungen, Abh. Math. Sem. Univ. Hamburg 16 (1949), nos. 3-4, 1-28. MR 11, 437; 872.

2. M. Eichler, Eine Verallgemeinerung der Abelschen Integrale, Math. Z. 67 (1957), 267-298. MR 19, 740.

3. —_ Grenzkreisgruppen und kettenbruchartige Algorithmen, Acta Arith. 11 (1965), 169-180. MR 37 \#4016.

4. - Lectures on modular correspondences, Tata Institute of Fundamental Research, Bombay, 1955/56.

5. R. C. Gunning, The Eichler cohomology groups and automorphic forms, Trans. Amer. Math. Soc. 100 (1961) 44-62. MR 24 \#A1136.

6. S. Y. Husseini and M. I. Knopp, Eichler cohomology and automorphic forms, Illinois J. Math. 15 (1971), 565-577. MR 44 \#2950. 
7. M. I. Knopp, Construction of automorphic forms on H-groups and supplementary Fourier series, Trans. Amer. Math. Soc. 103 (1962), 168-188. MR 25 \#197.

8. - - Notes on automorphic functions: An entire automorphic form of positive dimension is zero, J. Res. Nat. Bur. Standards Sect. B 71B (1967), 167-169. MR 38 \#6064.

9. M. I. Knopp, J. Lehner and M. Newman. A bounded automorphic form of dimension zero is constant, Duke Math. J. 32 (1965), 457-460. MR 33 \#4268.

10. I. Kra, Automorphic forms and kleinian groups, Benjamin, New York, 1972.

11. - - On cohomology of kleinian groups, Ann. of Math. (2) 89 (1969), 533-556. MR 41 \#8656a.

12. - On cohomology of kleinian groups. II, Ann. of Math. (2) 90 (1969), 576-590. MR 41 \#8656b.

13. J. Lehner, Automorphic integrals with preassigned period polynomials and the Eichler cohomology, Computers in Number Theory, Proc. Science Research Council Atlas Sympos. no. 2 (edited by A. O. L. Atkin and B. J. Birch), Academic Press, London and New York, 1971, pp. 49-56.

14. _ , Automorphic integrals with preassigned periods, J. Res. Nat. Bur. Standards Sect. B 73B (1969), 153-161. MR 40 \#1339.

15. - Discontinuous groups and automorphic functions, Math Surveys, no. 8, Amer. Math. Soc., Providence, R.I., 1964. MR 29 \#1332.

16. — The Eichler cohomology of a kleinian group, Math. Ann. 192 (1971), 125-143. MR 45 \#3704.

17. - A short course in automorphic functions, Holt, Rinehart and Winston, New York, 1966. MR 34 \#1519.

18. D. P. Niebur, Automorphic integrals of arbitrary positive dimension and Poincaré series, Doctoral Dissertation, University of Wisconsin, Madison, Wis., 1968.

19. H. Petersson, Automorphe Formen als metrische Invarianten. I. Automorphe Formen, metrische Verknüpfung, Eigenfunktionen linearer Funktionale, Math. Nachr. 1 (1948), 158-212. MR 10, 365.

20. - Automorphe Formen als metrische Invarianten. II. Multiplikative Differentiale als Grenzwerte metrischer Invarianten non stetig veränderlicher reeller Dimension, Math. Nachr. 1 (1948), 218-257. MR 10, 525.

21. - Explizite Konstruction der automorphen Orthogonalfunktionen in den multiplikativen Differentialklassen, Math. Nachr. 16 (1957), 343-368. MR 20 \#4643.

22. - Konstruktion der Modulformen und zu gewissen Grenzkreisgruppen gehörigen automorphen Formen von positiver reeller Dimension und die vollständige Bestimmung ihrer Fourier koeffizienten, S.-B. Heidelberger Akad. Wiss. Math.-Nat. K1. 1950, 417-494. MR 12, 806.

23. —, Zur analytischen Theorie der Grenzkreisgruppen. II, Math. Ann. 115 (1937/38), 175-204.

Mathematical Institute, University of Basel, Basel, Switzerland

Department of Mathematics, University of Wisconsin, Madison, Wisconsin 53706

Department of Mathematics, University of Illinois at Chicago Circle, Chicago, Illinots 60680 